\title{
OPEN Nuclear and cytosolic pS727-STAT3 levels correlate with overall survival of patients affected by clear cell renal cell carcinoma (ccRCC)
}

\author{
Jazmine Arévalo ${ }^{1}$, David Lorente ${ }^{2}$, Enrique Trilla ${ }^{2}$, María Teresa Salcedo $^{3}$, Juan Morote ${ }^{2}$ \& \\ Anna Meseguer ${ }^{1,4} \bowtie$
}

Clear cell renal cell carcinoma (ccRCC) is the most frequent and aggressive subtype of renal carcinoma. So far, the basis of its oncogenesis remains unclear resulting in a deficiency of usable and reliable biomarkers for its clinical management. Previously, we showed that nuclear expression of the signal transducer and activator of transcription 3 (STAT3), phosphorylated at its serine 727 (pS727), was inversely proportional to the overall survival of ccRCC patients. Therefore, in the present study, we validated the value of pS727-STAT3 as a clinically relevant biomarker in cCRCC. This work is a retrospective study on 82 ccRCC patients treated with nephrectomy and followed-up for 10 years. Immunohistochemical expression of pS727-STAT3 was analyzed on a tissue microarray and nuclear and cytosolic levels were correlated with clinical outcome of patients. Our results showed that pS727-STAT3 levels, whether in the nucleus $(p=0.002 ; 95 \% \mathrm{Cl} 1.004-1.026)$ or the cytosol $(p=0.040$; $95 \% \mathrm{Cl} 1.003-1.042$ ), significantly correlate with patients' survival in an independent-manner of clinicopathological features (Fuhrman grade, risk group, and tumor size). Moreover, we report that patients with high pS727-STAT3 levels who undergone adjuvant therapy exhibited a significant stabilization of the disease ( 20 months), indicating that pS727-STAT3 can pinpoint a subset of patients susceptible to respond well to treatment. In summary, we demonstrated that high pS727STAT3 levels (regardless of their cellular location) correlate with low overall survival of ccRCC patients, and we suggested the use of pS727-STAT3 as a prognostic biomarker to select patients for adjuvant treatment to increase their survival.

Clear cell renal cell carcinoma (ccRCC) is the most prevalent and aggressive histological subtype of renal cell carcinoma (RCC) accounting for $80-90 \%$ of all malignancies in adult kidney ${ }^{1,2}$. To date, ccRCC is considered the most lethal urological cancer due to its asymptomatic phenotype together with its resistance to chemotherapy and radiotherapy ${ }^{3,4}$. If ccRCC is detected in early stages, partial or radical nephrectomy is the first-line treatment and can prolong survival in $65 \%$ of patients. However, $30 \%$ of initial organ-confined tumors treated by surgical resection develop local recurrence or metastasis during the follow-up. Conversely, if detection occurs in advanced metastatic stage, there are very limited and no completely effective treatments to date ${ }^{5-7}$. Furthermore, adjuvant therapy has only been approved for metastatic patients, yet less than $50 \%$ of cases respond to treatment likely because of the underlying molecular heterogeneity among tumors ${ }^{8}$.

At present, prognosis after surgery has been modestly successful. Classic clinicopathologic features such as Fuhrman grade and tumor-node-metastasis (TNM) stage are the usual predictors of clinical outcome ${ }^{9,10}$, and despite they provide significant information, they are not accurate enough to predict disease progression by themselves. So far, although numerous and extensive studies on the genetic and biochemical features of ccRCC have been performed, there are few usable markers only related to treatment outcome ${ }^{11}$. This scenario poses the urgent need to identify specific biomarkers that can be used for early diagnosis, prognosis and to develop treatment strategies for all ccRCC patients.

\footnotetext{
${ }^{1}$ Renal Physiopathology CIBBIM-Nanomedicine, Vall d'Hebron Institut de Recerca (VHIR), Vall d'Hebron 119-129, 08035 Barcelona, Spain. ${ }^{2} U$ rology Department, Hospital Universitari Vall d'Hebron (HUVH), Barcelona, Spain. ${ }^{3}$ Pathology Department, Hospital Universitari Vall d'Hebron (HUVH), Barcelona, Spain. ${ }^{4}$ Biochemistry and Molecular Biology Department, Universitat Autònoma de Barcelona (UAB), Barcelona, Spain. ${ }^{\square}$ email: ana.meseguer@vhir.org
} 
In this context, previous studies from our group brought to light the role of the signal transducer and activator of transcription 3 (STAT3) in ccRCC by demonstrating that its activation resulted from the overexpression of the human hepatitis A virus cellular receptor 1 (hHAVCR1), also known as kidney injury molecule 1 $(\mathrm{KIM} 1)^{12}$. STAT3 is a ubiquitous transcription factor that regulates the expression of hundreds of genes involved in essential biological processes, thus, it is not surprising that its aberrant activation has been related to the onset of more than $50 \%$ of all human cancers ${ }^{13}$. Classically, STAT3 activation relies on the phosphorylation of its tyrosine $705(Y 705)^{14}$, nonetheless, recent reports described the phosphorylation of its serine 727 (S727) as a novel and non-canonical mechanism of STAT3 activation ${ }^{15,16}$. Previously, and in order to establish the role of STAT3 in the ccRCC, our group analyzed the expression of both phosphorylated residues (pY705 and pS727) on localized tumor samples from 98 ccRCC patients who had not undergone chemo- or immunotherapy before or after nephrectomy. Results from that study showed that only nuclear pS727-STAT3 -non pY705- levels were associated with the clinical outcome of ccRCC patients, therefore, it was proposed as an independent factor of overall survival ${ }^{12}$.

In the present study, we evaluated the prognostic value of pS727-STAT3 on a tissue microarray (TMA) from a new cohort of $82 \mathrm{ccRCC}$ patients followed-up for 10 years, and we correlated its expression levels, both in the nucleus and in the cytosol, with the clinical outcome of ccRCC patients. Moreover, the major difference from our previous patients' cohort ${ }^{12}$ was the inclusion of individuals who underwent tyrosine kinase inhibitors (TKIs) treatment after nephrectomy. These patients allowed the evaluation of the pS727-STAT3 potential to discriminate between those with different responses to adjuvant therapy.

\section{Results}

Study group. The present study included 56 men (68.3\%) and 26 women (31.7\%), median age: 72 years range: $38-92$, with ccRCC. The tumor was on the right side in 43 cases (52.4\%) and on the left side in 39 cases (47.6\%). A total of 77 patients (93.9\%) presented with localized tumors and $5(6.1 \%)$ with metastasis. Sixtyeight patients $(82.9 \%)$ underwent radical nephrectomy, while $14(17.1 \%)$ underwent nephron-sparing surgery (tumorectomy or partial nephrectomy). Fuhrman grade grouped I-II consisted of 42 cases (51.2\%), and III-IV consisted of 40 cases $(48.8 \%)$. The most frequently observed tumor size (pT) stages were pT1a in $45.1 \%$ and pT1b in $23.2 \%$. Lymphovascular invasion was present in only $3.6 \%$ of patients, while the risk group was low in $43.9 \%$, intermediate in $34.1 \%$, and high in $22 \%$ of patients studied. Finally, 15 patients $(18.3 \%)$ who recidivated after surgery, underwent tyrosine kinase inhibitors (TKIs) treatment (Table 1). All patients were considered for statistical analysis. Univariate analysis for cancer-specific survival following kidney surgery was statistically significant for the Fuhrman grade (I-II vs III-IV, $p=0.002$ ); risk group (low vs high, $p=0.006$ ); and tumor size (1-2 N0 M0 vs 3-4 N1,2 M1, $p=0.001$ ) (Table 2).

cCRCC tumors exhibit different pS727-STAT3 location patterns between the nucleus and the cytosol. Immunohistochemical analyses showed that unaffected adjacent tissue was negative for pS727STAT3 staining (Fig. 1, left panel), whereas ccRCC tumors exhibited positive staining both in the nucleus and the cytosol (Fig. 1, right panel). The threshold to distinguish patients with high or low pS727-STAT3 expression was determined as the median value based on the percentage and intensity of stained cells (H-score), thus, cutoff values were set at 80 and 40 (nucleus and cytosol, respectively). According to the H-score, 44 tumors (53.6\%) exhibited strong nuclear staining whilst 41 tumors (50\%) showed intense cytosolic staining. Conventionally, an activated STAT3, either by Y705 or S727 phosphorylation, is predominantly located within the nucleus regulating gene expression; however, recent studies have demonstrated that STAT3 phosphorylated at its S727 also exerts non-genomic functions in the mitochondria and the endoplasmic reticulum (ER $)^{16}$. These latest findings might explain the presence of pS727-STAT3 in both compartments observed in our study. More interestingly, different expression patterns were also observed among ccRCC tumors where pS727-STAT3 was i) only present in the nucleus (Fig. 1a), ii) completely cytosolic (Fig. 1b), or iii) simultaneously detected in both cellular compartments (Fig. 1c) but without a significant correlation between their independent H-scores $(p=0.2)$. The frequency of these expression patterns is summarized in Table 3. These results suggest that different pS727-STAT3 location patterns could determine different STAT3 functions during ccRCC development and progression.

High nuclear and cytosolic pS727-STAT3 levels identify patients with the lowest overall survival among those with advanced-stage ccRCC disease. Upon signal intensity evaluation using the $\mathrm{H}$-score, levels of pS727-STAT3 were correlated to the clinical outcome of ccRCC patients. Kaplan-Meier estimates of mortality (120 months overall survival) showed statistically significant differences in overall survival rates between patients in the same advanced-stage clinicopathologic group with high versus low pS727-STAT3 H-score: Fuhrman grade III-IV (nuclear $p=0.005$, cytosolic $p=0.027$ ), high-risk group (nuclear $p=0.018$, cytosolic $p=0.005$ ), and tumor size pT3-4 N1,2 M1 (nuclear $p=0.009$, cytosolic $p=0.012$ ) (Fig. 2a,b). Moreover, multivariate analysis for nuclear $(p=0.002 ; 95 \%$ CI $1.004-1.026)$ and cytosolic $(p=0.040 ; 95 \%$ CI $1.003-1.042)$ pS727-STAT3 H-score indicates that its presence-regardless of its location-significantly correlates with patient survival though cytosolic levels were not statistically as relevant as those in the nucleus (Table 4). Overall, these results demonstrate that high pS727-STAT3 levels-either in the nucleus or in the cytosol-represent a prognostic factor of low overall survival in advanced-stage ccRCC patients independently of the clinicopathological parameters.

CCRCC patients with high nuclear expression of pS727-STAT3 exhibit increased disease-free survival after adjuvant treatment. In the present study, patients who received adjuvant treatment $(n=15)$ in response to recurrence after surgery were found included in the high nuclear pS727-STAT3 levels 


\begin{tabular}{|c|c|}
\hline Variable & No. (\%) or variable unit \\
\hline \multicolumn{2}{|l|}{ Patient age (years) } \\
\hline Median & 72.17 \\
\hline Mean (range) & $70.56(38-92)$ \\
\hline \multicolumn{2}{|l|}{ Sex, No. (\%) } \\
\hline Male & $56(68.3)$ \\
\hline Female & $26(31.7)$ \\
\hline \multicolumn{2}{|l|}{ Tumor side } \\
\hline Right & $43(52.4)$ \\
\hline Left & $39(47.6)$ \\
\hline \multicolumn{2}{|l|}{ Clinical presentation, No. (\%) } \\
\hline Radical & $68(82.9)$ \\
\hline Neph. sparing & $14(17.1)$ \\
\hline Adjuvant treatment, No. (\%) & $15(18.3)$ \\
\hline \multicolumn{2}{|l|}{ Fuhrman grade, No. (\%) } \\
\hline I-II & $42(51.2)$ \\
\hline III-IV & $40(48.8)$ \\
\hline \multicolumn{2}{|l|}{ Primary tumor size, No. (\%) } \\
\hline pT1a & $37(45.1)$ \\
\hline pTlb & $19(23.2)$ \\
\hline pT2 & $4(4.9)$ \\
\hline pT3a & $13(15.9)$ \\
\hline pT3b & $8(9.8)$ \\
\hline pT4 & $1(1.2)$ \\
\hline \multicolumn{2}{|c|}{ Lymphovascular invasion, No. (\%) } \\
\hline $\mathrm{pNx}$ & $20(24.4)$ \\
\hline pN0 & $59(72.0)$ \\
\hline $\mathrm{pN1}$ & $2(2.4)$ \\
\hline $\mathrm{pN} 2$ & $1(1.2)$ \\
\hline \multicolumn{2}{|l|}{ Metastasis, No. (\%) } \\
\hline pM0 & 77 (93.9) \\
\hline pM1 & $5(6.1)$ \\
\hline \multicolumn{2}{|l|}{ Risk group, No. (\%) } \\
\hline Low & $36(43.9)$ \\
\hline Intermediate & $28(34.1)$ \\
\hline High & $18(22.0)$ \\
\hline \multicolumn{2}{|l|}{ Follow-up (months) } \\
\hline Median for all patients & 79.57 \\
\hline Mean for all patients & $72.15(2-112)$ \\
\hline
\end{tabular}

Table 1. Patients and tumor characteristics. Neph. sparing nephron-sparing surgery, $p T$ tumor size, $p N$ nodal involvement, $p M$ metastasis.

\begin{tabular}{|l|l|l|}
\hline Variable (grouped) & Univariate analysis mean $(95 \%$ CI) & $p$ value \\
\hline Fuhrman grade & $105.9(102.5-109.2)$ & \\
\hline I-II versus & $88.3(77.6-99.0)$ & 0.002 \\
\hline III-IV & $101.0(94.5-107.4)$ & \\
\hline Risk group & $91.7(80.8-102.5)$ & 0.006 \\
\hline Low versus & $105.0(101.2-108.8)$ & \\
\hline High & $79.0(63.6-94.5)$ & 0.001 \\
\hline Primary tumor size (pT) & & \\
\hline $1-2$ N0 M0 versus & & \\
\hline 3-4 N1,2 M1 &
\end{tabular}

Table 2. Cancer-specific survival following renal surgery. 

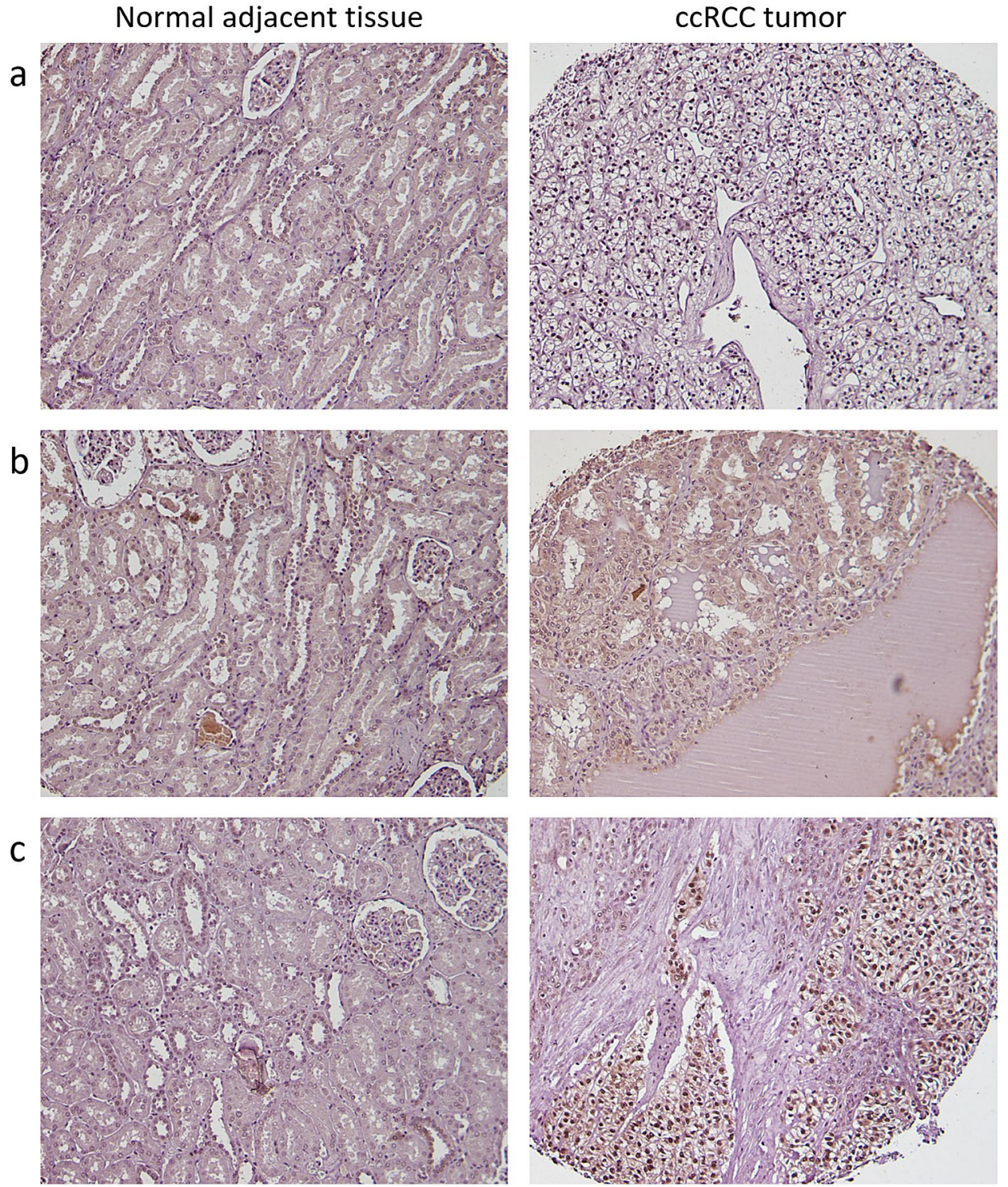

Figure 1. pS727-STAT3 location patterns in ccRCC tumors. Representative images of pS727-STAT3 immunohistochemical analysis showing normal counterparts (left panel) completely negative and tumor samples (right panel) exhibiting positive staining arranged in different location patterns: (a) only nuclear pS727STAT3; (b) only cytosolic pS727-STAT3, and (c) nuclear and cytosolic pS727-STAT3. Original magnification $\times 200$.

\begin{tabular}{|l|l|l|}
\hline pS727-STAT3 & H-score & $\boldsymbol{n}$ \\
\hline Only nuclear & $\geq 80$ & $24(29.2 \%)$ \\
\hline Only cytosolic & $\geq 40$ & $21(25.6 \%)$ \\
\hline Nuclear and cytosolic & $\geq 80, \geq 40$ & $20(24.3 \%)$ \\
\hline
\end{tabular}

Table 3. Incidence of different pS727-STAT3 expression patterns in ccRCC tumors.

group $(\mathrm{H}$-score $\leq 80)$ and advanced stages of the disease according to Fuhrman grade III-IV and tumor size pT3-4, N1,2 M1. Interestingly, the inclusion of these patients in our regression model resulted in a crossover of Kaplan-Meier curves, as observed at the top of the corresponding graphs (Fig. 2a). This particular observation indicates that these patients improved disease-free survival ( $\sim 20$ months $)$ after TKIs treatment, and suggests that pS727-STAT3 detection could also be used as a prognostic biomarker for the stratification of patients by 
a
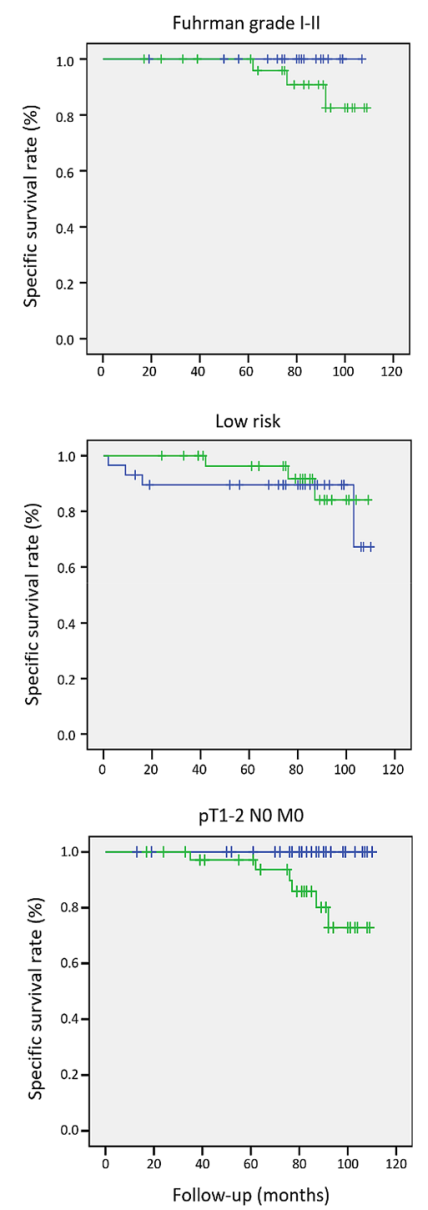

NUCLEUS
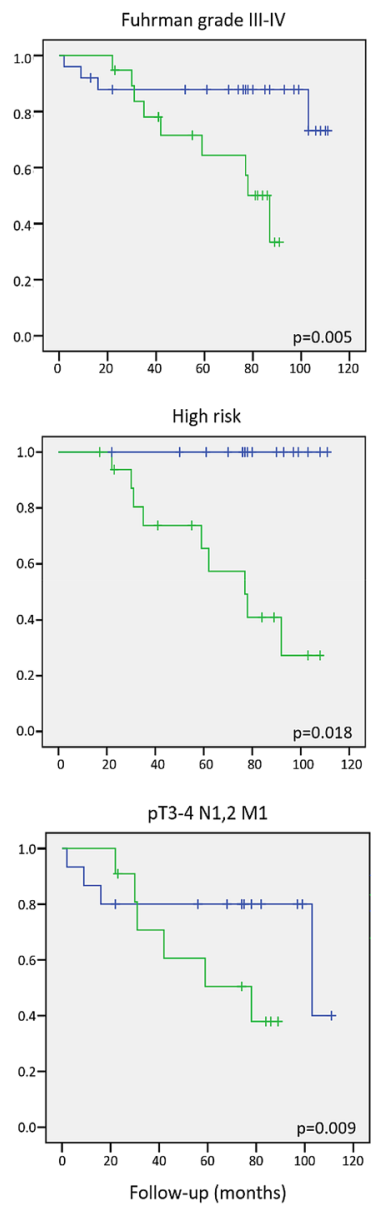

b

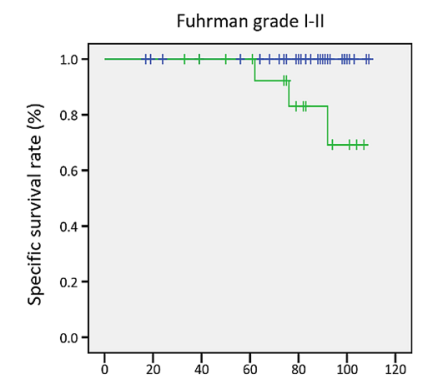

CYTOSOL
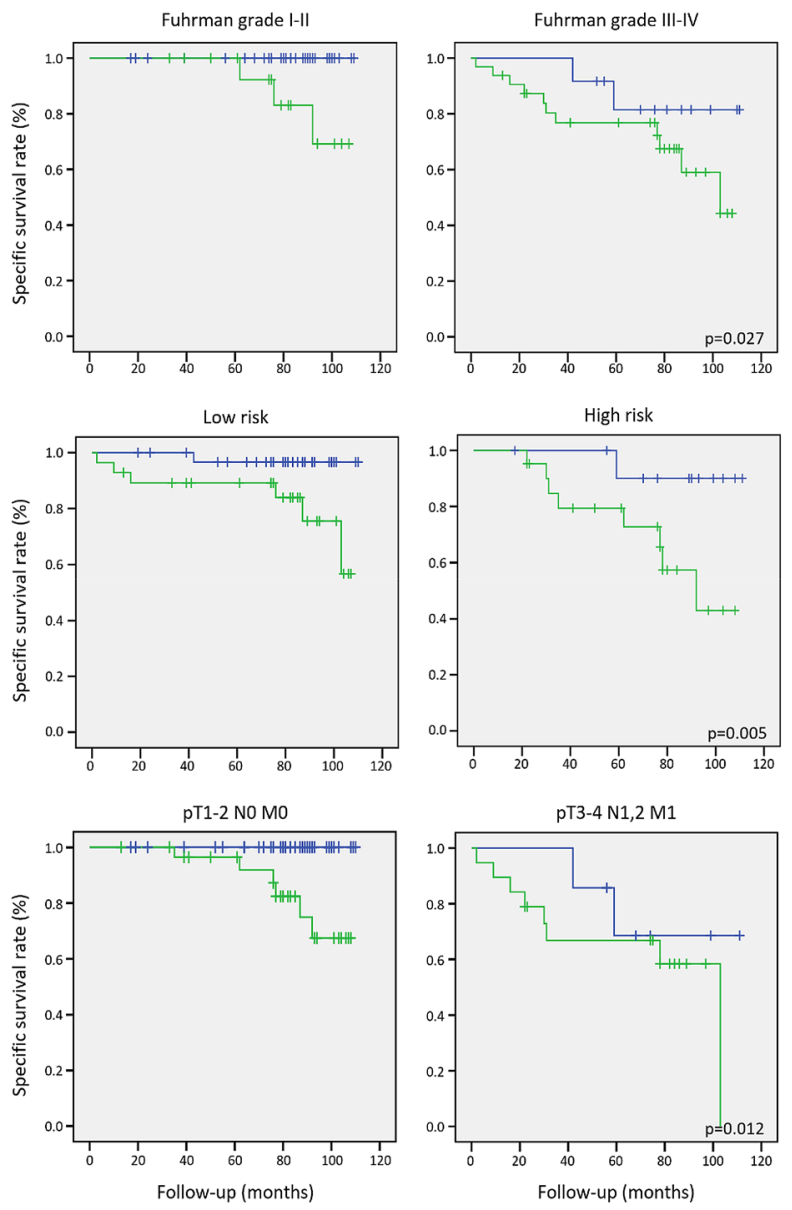

Figure 2. Correlation of pS727-STAT3 H-score with clinical outcome. Kaplan-Meier estimates of 120 months overall survival showing that patients with high pS727-STAT3 H-score (green lines) are prone to die earlier over time when compared to low pS727-STAT3 H-score patients (blue lines), especially those in advanced stages of the disease (Fuhrman grade III-IV, high risk, and tumor size pT3-4 N1,2 M1). Nuclear (blue line, HS $<80$; green line, HS $\geq 80$ ) (left panel) and cytosolic (blue line, HS < 40; green line, HS $\geq 40$ ) (right panel) pS727-STAT3 $\mathrm{H}$-score expression levels correlated to mean survival of ccRCC patients according to (a) Fuhrman grade (I-II, III-IV), (b) risk group (low, high), and (c) tumor size (pT1-2 N0 M0, pT3-4 N1,2 M1).

\begin{tabular}{|l|l|l|}
\hline H-score & Mean survival (95\% CI) (months) & $p$ value \\
\hline Nuclear pS727-STAT3 & $107.9(103.7-112.0)$ & \\
\hline$<80$ & $88.4(79.3-97.6)$ & 0.002 \\
\hline$\geq 80$ & $103.0(95.4-110.7)$ & \\
\hline Cytosolic pS727-STAT3 & $92.9(85.0-100.9)$ & 0.040 \\
\hline$<40$ & $\geq 40$ &
\end{tabular}

Table 4. Overall survival of ccRCC patients considering the expression of pS727-STAT3.

indicating which ones (those with high nuclear pS727-STAT3 levels) are susceptible to respond to adjuvant treatment and improve overall survival.

\section{Discussion}

Although multiple risk factors have been associated with the development of $\mathrm{cRCC}^{17}$, to date, the molecular mechanisms behind its etiology are not completely understood which leads to a lack of effective biomarkers clinically usable to predict disease progression or to select patients for particular therapies. So far, the most promising biomarkers for ccRCC are associated with treatment outcome, ranging from clinical parameters 
and endogenous substances (such as blood pressure or plasma proteins) to pathobiological features specific to individual tumors (such as mutations) ${ }^{11}$. Therefore, the study of molecular markers associated with ccRCC development and progression becomes urgent to provide a better understanding of the molecular pathogenesis of the disease, and consequently, its clinical outcome. In that scenario, a previous study from our group pointed out the role of STAT3 in ccRCC by demonstrating, for the first time, that nuclear presence of its phosphorylated form (at S727) correlated with the overall survival of ccRCC patients ${ }^{12}$. Accordingly, over the past years, it has been extensively confirmed that abnormal activation of STAT3 - either by Y705 or S727 phosphorylation-is a key event that contributes to oncogenesis in several tumors other than $\mathrm{CCRCC}^{13}$.

Here, we aimed to gain further insight into the value of pS727-STAT3 as a clinically relevant biomarker in ccRCC. Our results demonstrate that pS727-STAT3 levels constitute an independent prognostic factor of overall survival that divides advanced-stage ccRCC patients into two groups: those with low pS727-STAT3 levels and better prognosis, and those with high pS727-STAT3 levels prone to die earlier over time (Fig. 2a,b). Aligned with our results, other studies evaluating STAT3 activation (via pY705 or pS727) on tissue samples of other cancer types such as cervical intraepithelial neoplasia (CIN) and prostate cancer, have also reported a significant correlation of pS727-STAT3 levels to the clinical outcome of those patients ${ }^{18,19}$. These studies along with ours demonstrate that activation of STAT3 through S727 phosphorylation is indeed involved in the development of several malignancies, and suggest that pS727-STAT3 may be responsible for the expression of a subset of genes (different from those regulated by pY705) that promote a more aggressive tumoral phenotype.

Unlike our previous study, the present work included the analysis of cytosolic pS727-STAT3 levels due to recent findings indicating that STAT3 phosphorylation at S727 has an important role in the regulation of mitochondrial activity and modulation of $\mathrm{Ca}^{2+}$ release from the $\mathrm{ER}^{16}$; both processes in line with the pro-oncogenic role of STAT3. Several studies have demonstrated that mitochondrial STAT3 (mSTAT3), which refers to pS727STAT3, contributes to the maintenance of energy balance and cell survival under specific cellular stress conditions such as Ras-mediated transformation ${ }^{20}$. Specifically, pS727-STAT3 in the mitochondria has been related to an optimal electron transport chain (ECT) activity, an increase of membrane polarization and ATP production, and enhance of lactate dehydrogenase activity that, consequently, induces aerobic glycolysis and decreases reactive oxygen species (ROS) production ${ }^{21-23}$. In addition to these actions, protection from apoptosis is also triggered by inhibition of the mitochondrial permeability transition pore (MPTP) in collaboration with the $\mathrm{ER}^{24}$. In normal conditions, the regulation of $\mathrm{Ca}^{2+}$ homeostasis in the ER is mainly under the control of $\mathrm{Ca}^{2+}$ pumps and receptors activated by inositol 1,4,5-triphosphate $\left(\mathrm{IP}_{3}\right)$ called IP3 $\mathrm{R}^{25}$. This process takes place at the mitochondriaassociated membranes (MAMs), which locate at the interphase between the mitochondria and the $\mathrm{ER}^{26}$. As the membranes of both organelles are nearby, the excessive release of $\mathrm{Ca}^{2+}$ mediated by IP3R, in particular IP3R3, can lead to mitochondrial overload, which in turn triggers the opening of the MPTP and the initiation of the intrinsic apoptotic program ${ }^{27}$. Therefore, the ER-mitochondria space acts as a signaling hub for the activity of growth factors, oncogenes, and tumor suppressors that regulate IP3R3 activity ${ }^{16}$. Since both pY705- and pS727STAT3 localize to the ER and MAMs, phosphoablative mutants (Y705F and S727A) were used to evaluate their effect on IP3R3-mediated $\mathrm{Ca}^{2+}$ release founding that only pSer727-STAT3 inhibited $\mathrm{Ca}^{2+}$ release by interacting with IP3R3 and facilitating its degradation via the proteasome, thus, avoiding apoptosis ${ }^{26}$.

Our immunohistochemical analysis showed that ccRCC tumors exhibited three different location patterns of pS727-STAT3 between the nucleus and the cytosol. Specifically, pS727-STAT3 was found i) only in the nucleus, ii) only in the cytosol, or iii) in both compartments simultaneously; suggesting that pS727-STAT3 may exhibit different functions at certain stages of tumor development. It might be that pS727-STAT3 controls gene transcription in the nucleus, while regulates energy metabolism in the mitochondria and apoptosis through the ER. Moreover, the presence of pS727-STAT3 in the cytosol, specifically in the mitochondria, might respond to a shift in cellular energy metabolism driving the initial stages of transformation in agreement with the Warburg effect ${ }^{28}$. Otherwise, the presence of pS727-STAT3 in the ER could represent a well-established anti-apoptotic mechanism in the late stages of tumor development. Nevertheless, to confirm this hypothesis, more experiments should be performed to determine whether a correlation between pS727-STAT3 expression pattern and the different stages of ccRCC development actually exists. In line with our data, analysis of pS727-STAT3 levels in CIN samples has also identified nuclear and cytosolic expression of pS727-STAT3, both correlating with nuclear expression of the cellular marker of proliferation $\mathrm{Ki} 67^{18}$.

Besides the value of pS727-STAT3 as a prognostic factor of overall survival in ccRCC patients, our results also indicate that it may be useful as a novel biomarker to select patients who are candidates for adjuvant therapy. In our cohort, patients who received anti-angiogenic treatment (TKIs) corresponded to those exhibiting metastases or lymphovascular invasion at the time of surgery. In turn, none of the patients with localized tumors received any adjuvant treatment because no information regarding possible benefits for them was available until now. A recent study reported that adjuvant sunitinib versus placebo significantly improved the median duration of disease-free survival in locoregional tumors of ccRCC patients at high risk for tumor recurrence after nephrectomy ${ }^{29}$. Based on this study and our observation that ccRCC patients with a high pS727-STAT3 expression which undergone TKIs treatment experienced a significant stabilization of the disease ( 20 months $)$, we postulated that determination of pS727-STAT3 levels at the time of surgery could indicate that patients with localized tumors might benefit from adjuvant treatment versus those that only will suffer the adverse effects. We reasoned that the incorporation of molecular markers such as pS727-STAT3 into conventional models (such as Fuhrman grade and TNM stage) might enhance their prognostic accuracy. In that regard, we rationalized that patients in advanced stages of the disease with low pS727-STAT3 expression levels might not need adjuvant treatment because they do not recidivate; however, those patients in the high pS727-STAT3 group could benefit from the treatment. Although the number of patients with adjuvant therapy included in our cohort $(n=15)$ narrows the interpretation of our results and a larger study is needed, we propose the pS727-STAT3 as a putative biomarker able to indicate treatment after surgery in all advanced-stage ccRCC patients. 
In summary, the present study demonstrates that pS727-STAT3 expression, whether in the nucleus or the cytosol, correlates with a low overall specific cancer survival of ccRCC patients and represents an independent prognostic factor to classical clinicopathologic features. Moreover, we propose that measurement of pS727-STAT3 in tumor tissues at the time of surgery can determine which patients shall be treated with currently available adjuvant therapies to increase overall survival. Altogether, pS727-STAT3 represents a biomarker not only for the prognosis of the disease but also for the therapeutic stratification of ccRCC patients.

\section{Methods}

Case selection. Standard tumor data were obtained from 82 ccRCC patients treated with radical or partial nephrectomy between 2008 and 2010 and clinically followed up to 2017 at the Vall d'Hebron Hospital (Barcelona, Spain). Primary tumor histology was obtained from surgical resections and pathologic information was based on a re-review of all surgical samples to establish histologic subtype.

Clinical and pathologic information was retrospectively reviewed including date of diagnostic, demographics, tumor stage, Fuhrman grade, nodal and metastasis spread, initial and subsequent therapy, as well as outcome information. The anatomic extent of the tumors was classified using the TNM stage system, while prognostic stratification of ccRCC patients was scored using the University of California Los Angeles (UCLA) Integrated Staging System-Union Internationale Contre le Cancer (UICC) nomogram. Clinical and follow-up information was recorded by physician reports. Because of the availability of novel targeted therapies since 2008, patients who recidivated were treated with adjuvant therapy, nonetheless, all of them were included in the present study.

Tissue microarray (TMA). The TMA was constructed from paraffin-embedded samples of 82 ccRCC patients provided by the Department of Pathology at the Vall d'Hebron Hospital (Barcelona, Spain). One section from each specimen was stained with hematoxylin and eosin and reviewed for the selection of representative areas. For each sample, 3 representative areas of $1 \mathrm{~mm}$ diameter cores were obtained using a semi-automated tissue arrayer (Chemicon International). As controls, 3 cores of adjacent benign tissue far from the tumor were included. Finally, tissue cores were paraffin-embedded in a spaced array pattern ${ }^{30}$.

Informed consent from all participants was obtained for the use of all human tissues in this study following the rules of the Spanish Biomedical Research Law. The ethics committee of the Hospital Universitari Vall d'Hebron (HUVH) approved the study protocol and the methodology was performed following the relevant guidelines and regulations.

TMA immunohistochemistry. Four-micrometer-thick sections from TMA blocks were cut using a microtome, deparaffinized overnight $(\mathrm{ON})$ at $55^{\circ} \mathrm{C}$, and hydrated into milli- $\mathrm{Q} \mathrm{H}_{2} \mathrm{O}$ through sequential steps of graded ethanol. The immunohistochemistry (IHC) was performed using the EnVision + Dual Link SystemHRP (DAB+) kit (Dako \#K4065) according to the supplier's instructions. Antigen retrieval was performed with sodium citrate buffer pH 6.0 (Dako \#S2031) for $15 \mathrm{~min}$. Endogenous peroxidase activity was quenched followed by unspecific binding blocking with 5\% normal horse serum (Sigma Aldrich \# H0146) for $1 \mathrm{~h}$ at room temperature (RT). For pS727-STAT3 staining, TMAs were incubated ON at $4{ }^{\circ} \mathrm{C}$ with primary antibody a-pS727-STAT3 (Cell Signaling \#9134) at a final dilution of 1:50. The secondary antibody (labeled polymer-HRP mouse/rabbit) was incubated for $1 \mathrm{~h}$ at RT. Finally, HRP detection with DAB-Chromogen and hematoxylin (Sigma Aldrich \#HHS32) counterstaining were performed at RT for $20 \mathrm{~min}$ and $1 \mathrm{~min}$, respectively. As a negative control, the primary antibody was replaced with non-immune bovine serum (Alpha Diagnostics \# 20001-2-1).

TMA evaluation. pS727-STAT3 staining was scored by two renal pathology experts (MTS and ITR), blinded to clinicopathologic variables and fully independent to each other. pS727-STAT3 expression was evaluated in a semi-quantitative manner by immune-histo-score (H-score) based on the percentage and intensity of stained cells as follows: $0=$ no appreciable staining; $1=$ weak staining; $2=$ moderate staining; and $3=$ strong staining. Therefore, the $\mathrm{H}$-score was calculated as $1 \times(\%$ weak $)+2 \times(\%$ moderate $)+3 \times(\%$ intense $)$ ranging from 0 to 300 ${ }^{31}$. The inter-rater reliability between experts was substantial by having a Cohen's kappa of 0.75 for nuclear and 0.73 for cytosolic H-scores. For each ccRCC sample, 3 independent tissue cores were evaluated presenting an average coefficient of variance $(\mathrm{CV})$ of $23.4 \%$, therefore, $\mathrm{H}$-score was expressed as the mean.

Statistical analyses. Correlations between pS727-STAT3 expression and clinicopathologic parameters were evaluated with the nonparametric Mann-Whitney U test. Kaplan-Meier survival curves were compared using the log-rank test and multivariate analysis was carried out using a Cox regression model to estimate the independent prognostic importance of clinicopathologic parameters. Concordance between experts' scoring was determined using Cohen's kappa statistic ${ }^{32}$. Correlation between nuclear and cytosolic $\mathrm{H}$-scores was defined using linear regression, while the correlation between replicates was assessed by calculating the coefficient of variance among samples. All the statistical analysis was performed with the Statistical Package for Social Sciences software (SPSS, IBM).

\section{Data availability}

The datasets analyzed during the current study are not publicly available due to privacy policies. Data are however available from the corresponding author on reasonable request and with permission of the Spanish Biomedical Research Law and the ethics committee of the Hospital Universitari Vall d'Hebron (HUVH). 
Received: 23 November 2020; Accepted: 8 March 2021

Published online: 26 March 2021

\section{References}

1. Siegel, R. L., Miller, K. D. \& Jemal, A. Cancer statistics, 2018. CA. Cancer J. Clin. 68, 7-30 (2018).

2. Cohen, H. T. \& McGovern, F. J. Renal-cell carcinoma. N. Engl. J. Med. 353, 2477-2490 (2005).

3. Rini, B. I., Campbell, S. C. \& Escudier, B. Renal cell carcinoma. Lancet (London, England) 373, 1119-1132 (2009).

4. Ljungberg, B. et al. EAU guidelines on renal cell carcinoma: 2014 update. Eur. Urol. 67, 913-924 (2015).

5. Uchida, K. et al. Recurrence of renal cell carcinoma more than 5 years after nephrectomy. Int. J. Urol. 9, 19-23 (2002).

6. Levy, D. A., Slaton, J. W., Swanson, D. A. \& Dinney, C. P. Stage specific guidelines for surveillance after radical nephrectomy for local renal cell carcinoma. J. Urol. 159, 1163-1167 (1998).

7. Gong, J., Maia, M. C., Dizman, N., Govindarajan, A. \& Pal, S. K. Metastasis in renal cell carcinoma: Biology and implications for therapy. Asian J. Urol. 3, 286-292 (2016).

8. De Mulder, P. H. M. Targeted therapy in metastatic renal cell carcinoma. Ann. Oncol. Off. J. Eur. Soc. Med. Oncol. 18(Suppl 9), ix98-ix102 (2007).

9. Zigeuner, R. et al. External validation of the mayo clinic stage, size, grade, and necrosis (SSIGN) score for clear-cell renal cell carcinoma in a single European centre applying routine pathology. Eur. Urol. 57, 102-111 (2010).

10. Ficarra, V. et al. The 'Stage, Size, Grade and Necrosis' score is more accurate than the University of California Los Angeles Integrated Staging System for predicting cancer-specific survival in patients with clear cell renal cell carcinoma. BJU Int. 103, 165-170 (2009).

11. Hsieh, J. J. et al. Renal cell carcinoma. Nat. Rev. Dis. Prim. 3, 1-19 (2017).

12. Cuadros, T. et al. HAVCR/KIM-1 activates the IL-6/STAT-3 pathway in ccRCC and determines tumor progression and patient outcome. Cancer Res. 74(5), 1416-1428 (2014).

13. Guanizo, A. C., Fernando, C. D., Garama, D. J. \& Daniel, J. STAT3: A multifaceted oncoprotein. Growth Factors 36, 1-14 (2018).

14. Schindler, C., Levy, D. E. \& Decker, T. JAK-STAT signaling: From interferons to cytokines. J. Biol. Chem. 282, 20059-20063 (2007).

15. Aznar, S. et al. Simultaneous tyrosine and serine phosphorylation of STAT3 transcription factor is involved in RhoA GTPase oncogenic transformation. Mol. Biol. Cell 12, 3282-3294 (2001).

16. Avalle, L. \& Poli, V. Nucleus, mitochondrion, or reticulum? STAT3 à La Carte. Int. J. Mol. Sci. 19, 2820 (2018).

17. Karami, S. et al. Family history of cancer and renal cell cancer risk in Caucasians and African Americans. Br. J. Cancer 102, 1676-1680 (2010).

18. Yang, S. F. et al. The role of p-STAT3 (ser727) revealed by its association with Ki-67 in cervical intraepithelial neoplasia. Gynecol. Oncol. 98, 446-452 (2005).

19. Qin, H. R. et al. Activation of Stat 3 through a Phosphomimetic Serine727 Promotes Prostate Tumorigenesis Independent of Tyrosine705 phosphorylation. Cancer Res. 68, 7736-7741 (2010).

20. Gough, D. J. et al. Mitochondrial STAT3 supports RasDependent oncogenic transformation. Science (80-) 324, 1713-1716 (2009).

21. Tammineni, P. et al. The import of the transcription factor STAT3 into mitochondria depends on GRIM-19, a component of the electron transport chain. J. Biol. Chem. 288, 4723-4732 (2013).

22. Wegrzyn, J. et al. Function of mitochondrial Stat3 in cellular respiration. Science (80-) 323, 793-797 (2009).

23. Zhang, Q. et al. Mitochondrial localized Stat3 promotes breast cancer growth via phosphorylation of serine 727 . J. Biol. Chem. 288, 31280-31288 (2013).

24. Boengler, K., Hilfiker-Kleiner, D., Heusch, G. \& Schulz, R. Inhibition of permeability transition pore opening by mitochondrial STAT3 and its role in myocardial ischemia/reperfusion. Basic Res. Cardiol. 105, 771-785 (2010).

25. Mak, D. O. D. \& Foskett, J. K. Inositol 1,4,5-trisphosphate receptors in the endoplasmic reticulum: A single-channel point of view. Cell Calcium 58, 67-78 (2015).

26. Avalle, L. et al. STAT3 localizes to the ER, acting as a gatekeeper for ER-mitochondrion Ca 2+ fluxes and apoptotic responses. Cell Death Differ. 26, 932-942 (2019).

27. Morciano, G. et al. Molecular identity of the mitochondrial permeability transition pore and its role in ischemia-reperfusion injury. J. Mol. Cell. Cardiol. 78, 142-153 (2015).

28. Warburg, O. On the origin of cancer cells. Science (80-) 123, 309-314 (1956).

29. Motzer, R. J. et al. Adjuvant Sunitinib for high-risk renal cell carcinoma after nephrectomy: Subgroup analyses and updated overall survival results. Eur. Urol. 73, 62-68 (2018).

30. Skacel, M., Skilton, B., Pettay, J. D. \& Tubbs, R. R. Tissue microarrays: A powerful tool for high-throughput analysis of clinical specimens: A review of the method with validation data. Appl. Immunohistochem. Mol. Morphol. AIMM 10, 1-6 (2002).

31. Detre, S., Saclani Jotti, G. \& Dowsett, M. A 'quickscore' method for immunohistochemical semiquantitation: validation for oestrogen receptor in breast carcinomas. J. Clin. Pathol. 48, 876-878 (1995).

32. McHugh, M. L. Interrater reliability: The kappa statistic. Biochem. Medica 22, 276-282 (2012).

\section{Acknowledgements}

We would like to thank Dr. Cantero-Recasens for his helpful suggestions and critical reading of the manuscript.

\section{Author contributions}

J.A. designed the research, performed the experiments, managed and analyzed samples and datasets, interpreted results, wrote and revised the manuscript. A.M. conceived and designed the research, interpreted results of experiments, and edited and revised the manuscript. M.T.S. analyzed samples. D.L., E.T., and J.M. managed and analyzed datasets. All authors read and approved the final manuscript.

\section{Funding}

Funding was provided by Consejo Nacional de Ciencia y Tecnología, México (Grant No. 549678), Ministerio de Ciencia e Innovación (Grant No. SAF201459945-R), Red de Investigación Renal REDinREN (Grant No. 12/0021/0013) and Quality Mention from the Generalitat de Catalunya (Grant No. 2017 SGR).

\section{Competing interests}

The authors declare no competing interests.

Additional information

Correspondence and requests for materials should be addressed to A.M. 
Reprints and permissions information is available at www.nature.com/reprints.

Publisher's note Springer Nature remains neutral with regard to jurisdictional claims in published maps and institutional affiliations.

(c) (i) Open Access This article is licensed under a Creative Commons Attribution 4.0 International License, which permits use, sharing, adaptation, distribution and reproduction in any medium or format, as long as you give appropriate credit to the original author(s) and the source, provide a link to the Creative Commons licence, and indicate if changes were made. The images or other third party material in this article are included in the article's Creative Commons licence, unless indicated otherwise in a credit line to the material. If material is not included in the article's Creative Commons licence and your intended use is not permitted by statutory regulation or exceeds the permitted use, you will need to obtain permission directly from the copyright holder. To view a copy of this licence, visit http://creativecommons.org/licenses/by/4.0/.

(C) The Author(s) 2021 